\title{
Assessment of the learning curve of total thoracoscopic mitral valve repair using cumulative sum analysis (CUSUM)
}

\author{
Chao Song ${ }^{1}$, YunLong Fan ${ }^{1}$, Siming Zhu ${ }^{1}$, and Shengli Jiang ${ }^{2}$ \\ ${ }^{1}$ Medical School of Chinese PLA \\ ${ }^{2}$ Chinese PLA General Hospital
}

September 25, 2021

\begin{abstract}
Objective: To evaluate the learning curve and safety of total thoracoscopic mitral valve repair (MVP). Background: Total thoracoscopic MVP is characterized by minimal trauma, minimal bleeding, and short postoperative recovery time. The learning curve of any new procedure needs to be evaluated for learning and replication. However, minimally invasive mitral valve technique is a wide-ranging concept, no further analysis of the outcomes and learning curve of total thoracoscopic mitral valve repair has been performed. Methods: One hundred and fifty consecutive patients who underwent minimally invasive MVP alone without concurrent surgery were evaluated. Using Cardiopulmonary bypass (CPB) time and Aortic clamping (AC) time as evaluation variables, we visualized the learning curve for total thoracoscopic MVP using Cumulative sum analysis. We also analyzed important postoperative variables such as postoperative drainage, duration of mechanical ventilation, ICU stay and postoperative hospital stay. Results: The slope of the fitted curve was negative after 75 procedures, and the learning curve could be crossed after the completion of the 75th procedure when AC and CPB time were used as evaluation variables. And as the number of surgical cases increased, CPB, AC, postoperative drainage, duration of mechanical ventilation, ICU stay and postoperative hospital stay all showed different degrees of decrease. The incidence of postoperative adverse events is similar to conventional mitral valve repair. Conclusions: Compared to conventional MVP, total thoracoscopic MVP provides the same satisfactory surgical results and stabilization can be achieved gradually after completion of the 75th procedure.
\end{abstract}

Assessment of the learning curve of total thoracoscopic mitral valve repair using cumulative sum analysis (CUSUM)

Chao Song ${ }^{1,2}$, Yunlong Fan ${ }^{1,2}$, Siming Zhu ${ }^{1,2}$, Shengli Jiang ${ }^{2 *}$

${ }^{1}$ Medical School of Chinese PLA, Beijing, 100853, P.R. China

${ }^{2}$ Department of Cardiovascular Surgery, the First Medical Centre of Chinese PLA General Hospital, Beijing 100853, China

${ }^{*}$ Correspondence: Dr. Shengli Jiang, Department of Cardiovascular Surgery, Chinese PLA General Hospital, 100853, Beijing, China. E-mail: JiangSL301hospital@163.com.

Conflicts of interest

The authors declare that there are no conflicts of interest.

This research did not receive any specific grant from funding agencies in the public, commercial, or not-forprofit sectors.

Objective: To evaluate the learning curve and safety of total thoracoscopic mitral valve repair (MVP). 
Background: Total thoracoscopic MVP is characterized by minimal trauma, minimal bleeding, and short postoperative recovery time. The learning curve of any new procedure needs to be evaluated for learning and replication. However, minimally invasive mitral valve technique is a wide-ranging concept, no further analysis of the outcomes and learning curve of total thoracoscopic mitral valve repair has been performed.

Methods: One hundred and fifty consecutive patients who underwent minimally invasive MVP alone without concurrent surgery were evaluated. Using Cardiopulmonary bypass (CPB) time and Aortic clamping (AC) time as evaluation variables, we visualized the learning curve for total thoracoscopic MVP using $\mathrm{Cu}-$ mulative sum analysis. We also analyzed important postoperative variables such as postoperative drainage, duration of mechanical ventilation, ICU stay and postoperative hospital stay.

Results: The slope of the fitted curve was negative after 75 procedures, and the learning curve could be crossed after the completion of the 75th procedure when $\mathrm{AC}$ and $\mathrm{CPB}$ time were used as evaluation variables. And as the number of surgical cases increased, CPB, AC, postoperative drainage, duration of mechanical ventilation, ICU stay and postoperative hospital stay all showed different degrees of decrease. The incidence of postoperative adverse events is similar to conventional mitral valve repair.

Conclusions: Compared to conventional MVP, total thoracoscopic MVP provides the same satisfactory surgical results and stabilization can be achieved gradually after completion of the 75 th procedure.

\section{Introduction}

Minimally invasive cardiac surgery has become a new trend in modern medicine because of its reduced trauma, less bleeding and shorter post-operative recovery time ${ }^{1-3}$, and there has been a significant increase in the number of studies related to minimally invasive mitral valve surgery ${ }^{4-7}$. Since minimally invasive mitral valve surgery was first described by Cohn and Cosgrove in the mid-1990s, a variety of minimally invasive procedures, including the parasternal, hemi-sternotomy, mini-thoracotomy, totally robotic approach, and totally endoscopic approach, have been proposed ${ }^{8}$. Among them, Carpentier et al. successfully performed the first thoracoscopic-assisted mitral valve repair (MVP) in $1996^{9}$, and more and more medical centers are showing interest in thoracoscopic-assisted MVP. Using thoracoscopic techniques, the "direct view" of the valve can be clearly provided and the mitral valve and perivalvular structures can be better evaluated ${ }^{10}$. With the update of minimally invasive surgery technology, total thoracoscopic techniques have been widely recognized for its better affordability and less invasiveness compared to total robotic approaches and thoracoscopicassisted techniques ${ }^{11}$. However, to date, relatively few centers can carry out totally thoracoscopic MVP independently ${ }^{12}$. Increased postoperative complications, reduced repair quality and a longer learning curve are probably the main concerns regarding this technique. Minimally invasive mitral valve technique is a wide-ranging concept, yet there is no further subgroup analysis of its surgical outcomes and learning curve based on different procedures.

In this article, we summarize our clinical experience with 150 consecutive total thoracoscopic MVP and provide a comprehensive evaluation of the total thoracoscopic MVP technique using its CUSUM (cumulative summation) learning curve and early clinical outcomes, which in turn may help surgeons or centers in the early stages of learning the total thoracoscopic technique to improve the safety of the procedure and to benefit more patients.

\section{Materials and methods}

\section{Study design}

The research was conducted in accordance with the Declaration of Helsinki (revised 2013). The research was a retrospective clinical study without any specific interventions for the patients, all patients had previously approved the use of their medical records for research purposes, and the study protocol had been approved by local institutional review board.

\section{Patients}


From October 2017 to July 2021, a total of 150 total thoracoscopic MVP were performed by an experienced surgeon in the heart center of Chinese PLA General Hospital. In order to reduce the effect of simultaneous surgery on cardiopulmonary bypass (CPB) time, aortic clamping (AC) time, patients with concurrent aortic valve surgery, congenital heart surgery, radiofrequency ablation of atrial fibrillation, mucosal aneurysm resection, combined severe coronary artery disease, and low ejection fraction $(<30 \%)$ were excluded.

\section{Surgical technique}

After the induction of general anesthesia, a left-sided double-lumen endotracheal tube was placed to allow for single-lung ventilation and defibrillator pads was placed across the chest. The patient was placed in a $30^{\circ}$ left side position, a small pillow is placed under the scapula to open up the axillary space. A TEE probe was then placed to evaluate mitral valve and ventricular function in all patients before and after the procedure.

CPB was routinely established through the femoral artery, femoral vein, and right internal jugular vein. The main surgical hole (approximately $3 \mathrm{~cm}$ ) was located in the fourth intercostal space outside the right midclavicular line, through which the cardiac arrest fluid flushing tube was also passed. The thoracoscopy was placed through the third intercostal space on the right anterior axillary line, after inserting the aortic root cannula, while the left cardiac drainage and Chitwood clamp were punctured through the fourth intercostal space. At our center, MVP is performed using a conventional left atrial incision, parallel to the interatrial sulcus, and an artificial pneumothorax is routinely established using CO2.

\section{Data collection and analysis}

Preoperative baseline information, intraoperative CPB time and AC time, postoperative bleeding, and incidence of surgical adverse events, including the presence of delayed extubation, secondary intubation, pleural effusion, pneumothorax, atrial fibrillation, poor incision healing, and cerebral infarction, were collected by the same physician.

Cumulative sum (CUSUM) is a statistical method that focuses on results rather than on the process of performing a program skills, it generates graphs that allow for quick detection of deviations from preestablished standards and is an alternative tool that can be used to evaluate the performance of individual programs ${ }^{13}$. CUSUM can be generated based on set acceptable and unacceptable failure rates and the degree to which type $1(\alpha)$ and type $2(\beta)$ errors (false positive and false negative errors) will be tolerated ${ }^{14}$. CUSUM was defined as $\mathrm{Sn}=[?](\mathrm{Xi}-\mathrm{p} 0)$, where $\mathrm{Xi}=0$ for success and $\mathrm{Xi}=1$ for failure, $\mathrm{p} 0$ is the target reference ${ }^{15}$. In the CUSUM chart generated by Minitab Statistical Software (Version 20.3), surgical cases are arranged chronologically on the horizontal axis. The vertical axis $\mathrm{f}$ represents the cumulative total of AC time and CPB time, respectively. When the curve crosses the boundary, the complication rate of the surgeon is equal to or lower than the acceptable rate, which means that the learning curve is reached.

Perioperative data were analyzed using SPSS 26.0 software (SPSS Inc., Chicago, Illinois, USA). Continuous variables were expressed as means and standard deviations. Categorical variables were expressed as percentages. Continuous variables were compared using Student t-test and ANOVA analysis, and categorical variables were analyzed using chi-square test and Fischer's exact test. And the significant differences were defined at $\mathrm{p}<0.05$.

\section{Results}

\section{Learning curve}

All patients completed the surgery successfully, with no intermediate open-heart surgery and no perioperative deaths. Specific details of the baseline characteristics of the included patients are shown in Table 1. Operation and postoperative characteristics are summarized in Table 2. The learning curve of AC and CPB visualized with CUSUM diagrams are shown in Figure1. Each trend graph confirmed the presumed shape of an initially steep slope. The fitted equation for CPB is $\mathrm{Y}=192.5462959411959+-1.173480390520274$ * $\mathrm{x}$ $+0.01115565046303966 * \mathrm{x}^{2}+-4.161350192966322 \mathrm{e}-005 * \mathrm{x}^{3}, \mathrm{R}^{2}=0.434$, and the fitted equation for AC is $\mathrm{Y}=131.5939091152518+-0.9761575056383154 * \mathrm{x}+0.01005722184889189 * \mathrm{x}^{2}+-3.832091085504812 \mathrm{e}-005$ 
$* \mathrm{x}^{3}, \mathrm{R}^{2}=0.412$ (Figure2). As shown in the CUSUM plot, the slope of the fitted curve was negative after 75 procedures, so the learning curve could be crossed after completing 75 procedures. As the number of surgical cases increased, $\mathrm{CPB}, \mathrm{AC}$, postoperative drainage, duration of mechanical ventilation, ICU stay and postoperative hospital stay all showed different degrees of decrease, as shown in Figure3. This statistical analysis showed a gradual decrease in the important variables toward a positive results trend as the accumulated number of cases increased in time. Time series plots were generated for visualization of the postoperative data, and we divided the enrolled patients into three groups (50/per group) chronologically for better intergroup comparison. Compared to the other variables that showed statistically significant differences, ICU stay, although not showing statistically significant differences, also showed a moderate decrease.

\section{Postoperative outcome}

The overall postoperative adverse event incidence was $2.67 \%$, as shown in the Table3, none of the patients experienced hospital mortality, serious complications, respiratory failure, delayed extubation, secondary intubation, pleural effusion, pneumothorax, cerebral hemorrhage, pulmonary Infection, poor healing of incision and sternal dehiscence. IABP implantation was performed in two of the patients for transient decreased cardiac output, and the IABP was successfully removed. one patient underwent dialysis for renal insufficiency, and this patient had a preoperative history of renal insufficiency, and the intraoperative hypoperfusion may have exacerbated the renal impairment, one patient experienced brain infarction without disability.

\section{Discussion}

Since the first successful thoracoscopic mitral valve surgery performed by Carpentier et al, minimally invasive valve surgery has made great progress in the last 25 years, including inferior hemifacial incision, direct right mini-incision and thoracoscopic-assisted right mini-incision, and robotic-assisted mitral valve surgery, all of which have been shown to have reduced trauma, decreased bleeding, and better recovery compared to traditional MV surgery ${ }^{9}$. With similar repair results, total thoracoscopic MVP could also be used for complex mitral valve lesions with the simultaneous advantages of minimally invasive valve surgery ${ }^{16}$. MVP is a more demanding technique compared with mitral valve replacement ${ }^{17,18}$, which is why in the early development of thoracoscopic mitral valve repair, high postoperative complications and prolonged operative time led some researchers to be suspicious of this technique ${ }^{19}$.

When performing a new surgical procedure, surgeons need to understand the learning curve of the procedure in order to improve the learning efficiency of the procedure. By visualizing the learning curve, CUSUM realistically reflects the progression of a surgeon learning, practicing, and then plateauing for a new procedure, and eventually reaching stability. CUSUM could be used to provide continuous performance data and possibly to evaluate the training program themselves ${ }^{14}$. Since its first application in medical statistics by Bolsin S, CUSUM has been widely used in the evaluation of learning curves for various new procedures ${ }^{13,15}$. By repeating and updating the learning curve after accumulating the variables one by one, the change pattern of the learning curve can be identified quickly. The CUSUM curve showed an upward slope when the operation time exceeded the mean value, and conversely, the CUSUM curve showed a downward slope. In this study, the CUSUM learning curve underwent a significant upward trend before the 75th surgery, and significant improvements in both $\mathrm{AC}$ and $\mathrm{CPB}$ were observed after the 75th surgery, which was similar to the results of previous learning curve studies of minimally invasive valve surgery, Although there were significant differences between different studies, the overall tendency of change in CUSUM was similar, with the number of procedures required to overcome the learning curve ranging from 64 to $116^{20-22}$. However, learning curve outcomes may vary considerably between different minimally invasive valve procedures, with parasternal, hemi-sternotomy, and mini-thoracotomy approaches likely to reach plateau more rapidly, and correspondingly, totally robotic approaches, and totally endoscopic approach may require more time and training to achieve stability. However, previous studies related to learning curves of minimally invasive mitral valve repair have not performed more precise subgroup analyses according to the different procedures and did not make strict exclusions for simultaneous procedures, because any simultaneous procedure may result in prolonged AC and CPB times ${ }^{21-24}$. In this study, the CUSUM of postoperative adverse events could not be visualized due to the low incidence, but according to previous literature, the incidence of postope- 
rative complications was similar between total thoracoscopic MVP and conventional MVP surgery ${ }^{25}$. The CUSUM curves of AC and CPB showed a steep upward trend until the 26th surgery, followed by a slow upward and plateau period. This may be due to early re-familiarization and re-confirmation of the operative field and anatomy under fully thoracoscopic conditions as well as a slightly reduced level of cooperation, as fully thoracoscopic surgery may place higher demands on the assistants, anesthesiologists, and nursing team in addition to the surgeon, and each of these variables has its own learning curve. According to our clinical experience, an increasing number of patients requiring simultaneous surgery for tricuspid valve lesions, infective endocarditis, mucinous tumors, and atrial septal defects underwent total thoracoscopic MVP as the number of procedures increased, although we were unable to include patients with these concomitant procedures in the study given the homogeneity of the study population.

\section{Strengths and limitations}

The advantage of this study over previous studies is that we set strict inclusion and exclusion criteria, and patients who underwent concurrent surgery such as atrial septal defect repair and tricuspid valve repair were strictly excluded, which to some extent reduced the effect of concurrent surgery on the target variables. Secondly, we exclusively evaluated the learning curve of total thoracoscopic mitral valvuloplasty, dividing the minimally invasive valve techniques further in detail, for the difference in learning curve between different minimally invasive approaches can be substantial.

This study also has some limitations. Firstly, this study is a retrospective study, and the selection of patients undergoing total thoracoscopic MVP may have undergone operator intervention, which may have influenced the subsequent CUSUM curves to some extent. Second, because of objective limitations, we were unable to propensity-match the study population to select patients who underwent conventional MVP with similar clinical baseline characteristics as a dyadic group for the control study. Finally, our center is probably the largest total thoracoscopic cardiac surgery center in China, and the number of total thoracoscopic cardiac surgeries performed as a routine procedure in our center is more than 4 per week. Maintaining a certain degree of frequency of total thoracoscopic surgeries may have a non-negligible impact on the learning curve, however, this may receive some limitations in terms of replication in other centers due to objective conditions.

\section{Acknowledgments}

Funding: None.

\section{REFERENCES}

1. Casselman FP, Van Slycke S, Wellens F, et al. Mitral valve surgery can now routinely be performed endoscopically. Circulation . 2003;108 Suppl 1:II48-54. https://doi.org/10.1161/01.cir.0000087391.49121.ce

2. Schmitto JD, Mokashi SA, Cohn LH. Minimally-invasive valve surgery.J Am Coll Cardiol . 2010;56(6):455462. https://doi.org/10.1016/j.jacc.2010.03.053

3. Holzhey DM, Seeburger J, Misfeld M, Borger MA, Mohr FW. Learning minimally invasive mitral valve surgery: a cumulative sum sequential probability analysis of 3895 operations from a single high-volume center. Circulation . 2013;128(5):483-491. https://doi.org/10.1161/CIRCULATIONAHA.112.001402

4. Kastengren M, Svenarud P, Ahlsson A, Dalén M. Minimally invasive mitral valve surgery is associated with a low rate of complications. J Intern Med . 2019;286(6):614-626. https://doi.org/10.1111/joim.12974

5. Javadikasgari H, Suri RM, Tappuni B, Gillinov AM. Minimally invasive mitral valve repair. Heart . 2018;104(10):861-867. https://doi.org/10.1136/heartjnl-2016-310774

6. Harky A, Botezatu B, Kakar S, Ren M, Shirke MM, Pullan M. Mitral valve diseases: Pathophysiology and interventions. Prog Cardiovasc Dis . 2021. https://doi.org/10.1016/j.pcad.2021.03.008

7. Desai MY, Grigioni F, Di Eusanio M, et al. Outcomes in Degenerative Mitral Regurgitation: Current State-of-the Art and Future Directions.Prog Cardiovasc Dis . 2017;60(3):370-385. https://doi.org/10.1016/j.pcad.2017.10.005 
8. Marin Cuartas M, Javadikasgari H, Pfannmueller B, et al. Mitral valve repair: Robotic and other minimally invasive approaches. Prog Cardiovasc Dis . 2017;60(3):394-404. https://doi.org/10.1016/j.pcad.2017.11.002

9. Carpentier A, Loulmet D, Carpentier A, et al. [Open heart operation under videosurgery and minithoracotomy. First case (mitral valvuloplasty) operated with success]. C R Acad Sci III . 1996;319(3):219-223.

10. Cui H, Zhang L, Wei S, et al. Early clinical outcomes of thoracoscopic mitral valvuloplasty: a clinical experience of 100 consecutive cases. Cardiovasc Diagn Ther . 2020;10(4):841-848. https://doi.org/10.21037/cdt$20-440$

11. Sündermann SH, Czerny M, Falk V. Open vs. Minimally Invasive Mitral Valve Surgery: Surgical Technique, Indications and Results. Cardiovasc Eng Technol . 2015;6(2):160-166. https://doi.org/10.1007/s13239015-0210-5

12. Chiu KM, Chen RJ. Videoscope-assisted cardiac surgery. $J$ Thorac Dis . 2014;6(1):22-30. https://doi.org/10.3978/j.issn.2072-1439.2014.01.04

13. Bolsin S, Colson M. The use of the Cusum technique in the assessment of trainee competence in new procedures. Int J Qual Health Care . 2000;12(5):433-438. https://doi.org/10.1093/intqhe/12.5.433

14. Starkie T, Drake EJ. Assessment of procedural skills training and performance in anesthesia using cumulative sum analysis (cusum). Can J Anaesth . 2013;60(12):1228-1239. https://doi.org/10.1007/s12630013-0045-1

15. Rogers CA, Reeves BC, Caputo M, Ganesh JS, Bonser RS, Angelini GD. Control chart methods for monitoring cardiac surgical performance and their interpretation. $J$ Thorac Cardiovasc Surg . 2004;128(6):811-819. https://doi.org/10.1016/j.jtcvs.2004.03.011

16. Bergsland J, Mujanovic E, Elle OJ, Mirtaheri P, Fosse E. Minimally invasive repair of the mitral valve: technological and clinical developments. Minim Invasive Ther Allied Technol . 2011;20(2):72-77. https://doi.org/10.3109/13645706.2011.554843

17. Nishimura RA, Vahanian A, Eleid MF, Mack MJ. Mitral valve disease-current management and future challenges. Lancet . 2016;387(10025):1324-1334. https://doi.org/10.1016/S0140-6736(16)00558-4

18. Salvador L, Mirone S, Bianchini R, et al. A 20-year experience with mitral valve repair with artificial chordae in 608 patients. $J$ Thorac Cardiovasc Surg . 2008;135(6):1280-1287. https://doi.org/10.1016/j.jtcvs.2007.12.026

19. Welp H, Martens S. Minimally invasive mitral valve repair.Curr Opin Anaesthesiol . 2014;27(1):65-71. https://doi.org/10.1097/ACO.0000000000000038

20. Hayashi Y, Nakamura Y, Hirano T, Ito Y, Watanabe T. Cumulative sum analysis for the learning curve of minimally invasive mitral valve repair. Heart Vessels . 2021. https://doi.org/10.1007/s00380-021-01838-7

21. Dokollari A, Cameli M, Kalra DS, et al. Learning curve predictors for minimally invasive mitral valve surgery; how far should the rabbit hole go. J Card Surg . 2020;35(11):2934-2942. https://doi.org/10.1111/jocs.14939

22. Vo AT, Nguyen DH, Van Hoang S, et al. Learning curve in minimally invasive mitral valve surgery: a single-center experience. J Cardiothorac Surg . 2019;14(1):213. https://doi.org/10.1186/s13019-019-1038-0

23. Wu X, Wei W, He Y, Qin H, Qi F. Analysis of the Learning Curve in Mitral Valve Replacement Through the Right Anterolateral Minithoracotomy Approach: A Surgeon's Experience with the First 100 Patients.Heart Lung Circ . 2019;28(3):471-476. https://doi.org/10.1016/j.hlc.2018.02.002

24. Murzi M, Cerillo AG, Bevilacqua S, et al. Enhancing departmental quality control in minimally invasive mitral valve surgery: a single-institution experience. Eur J Cardiothorac Surg . 2012;42(3):500-506. https://doi.org/10.1093/ejcts/ezs050 
25. Davierwala PM, Seeburger J, Pfannmueller B, et al. Minimally invasive mitral valve surgery: "The Leipzig experience". Ann Cardiothorac Surg . 2013;2(6):744-750. https://doi.org/10.3978/j.issn.2225319X.2013.10.14

\section{Figure}
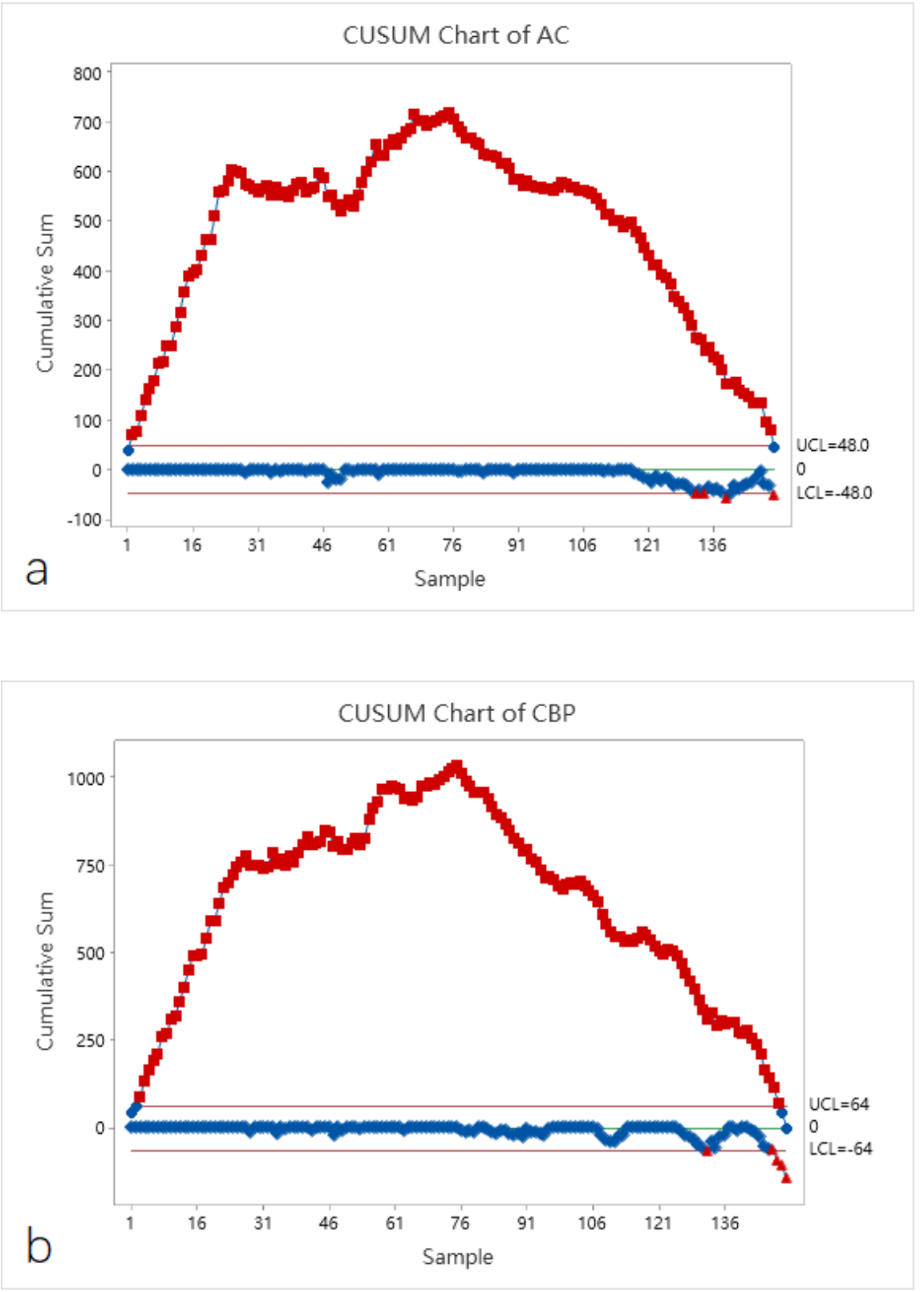

Figure1 CUSUM curve analyses of the AC time(a) and CPB time(b). 


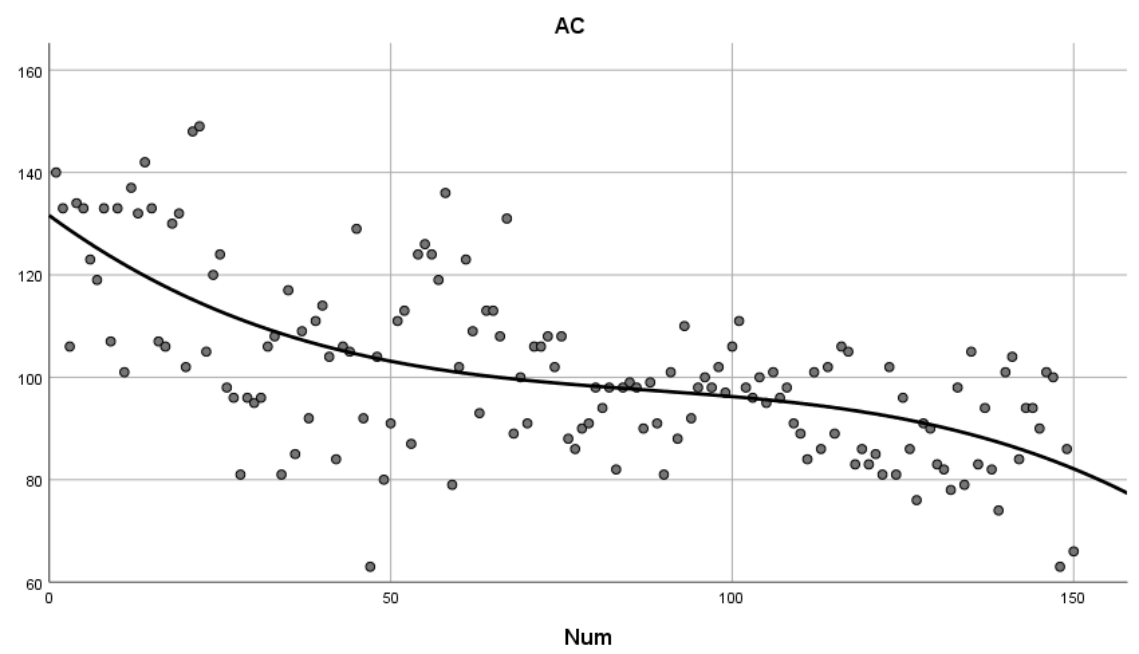

a

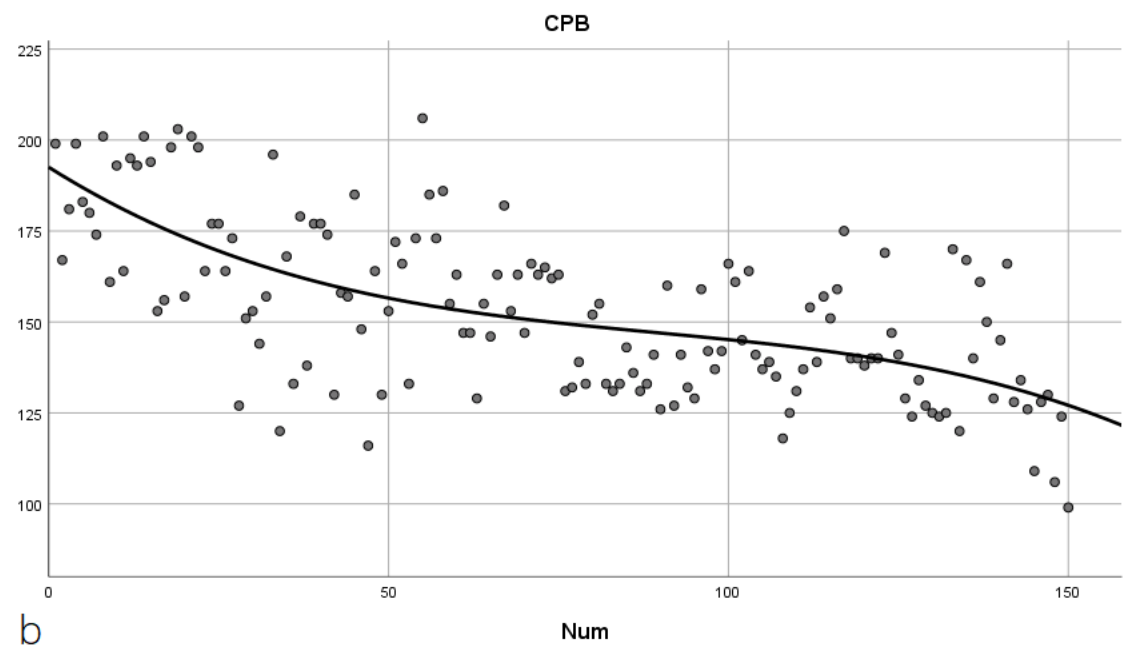

Figure2 Time series chart of $\mathrm{AC}(\mathrm{a})$ and $\mathrm{CPB}(\mathrm{b})$ time 

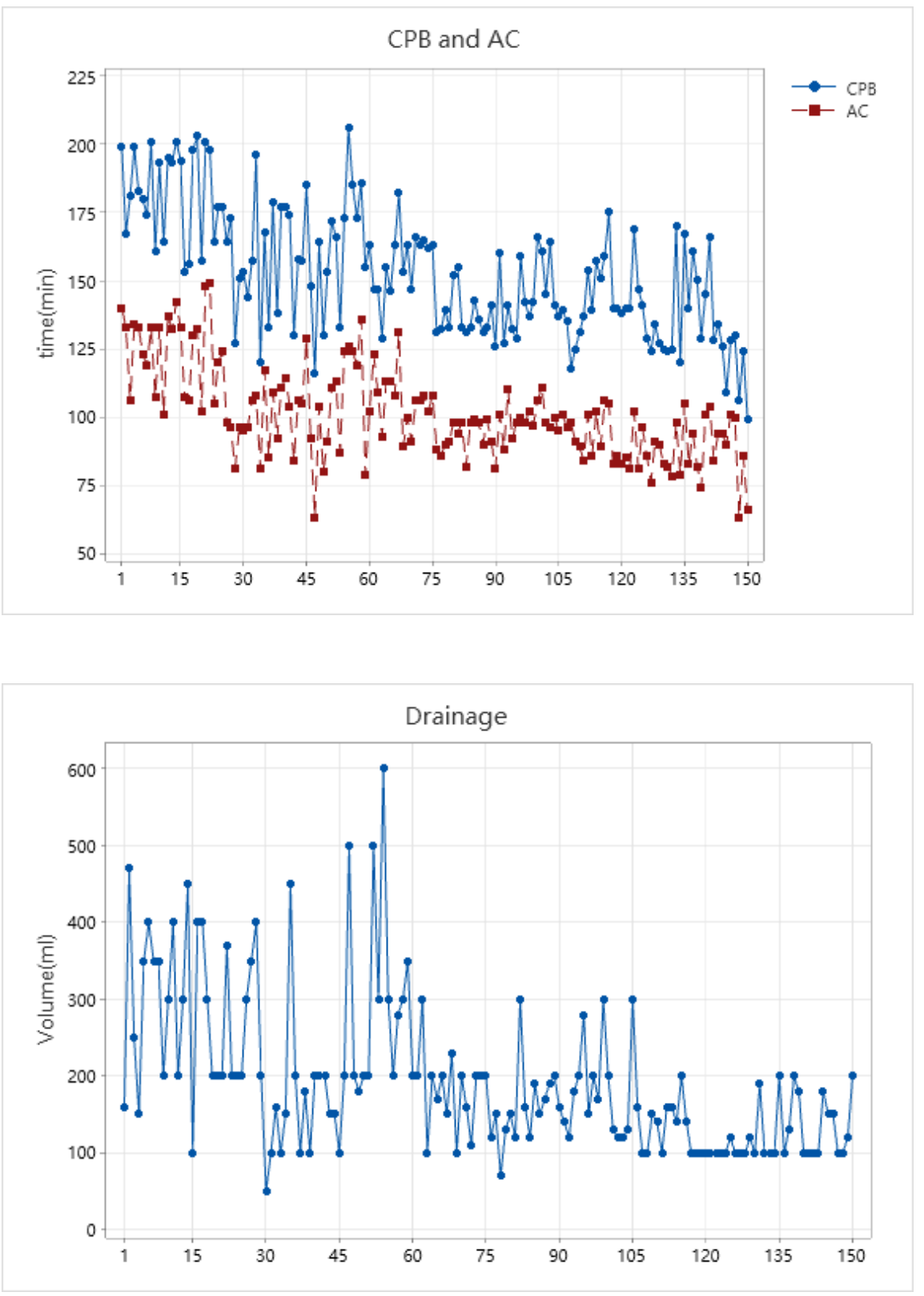

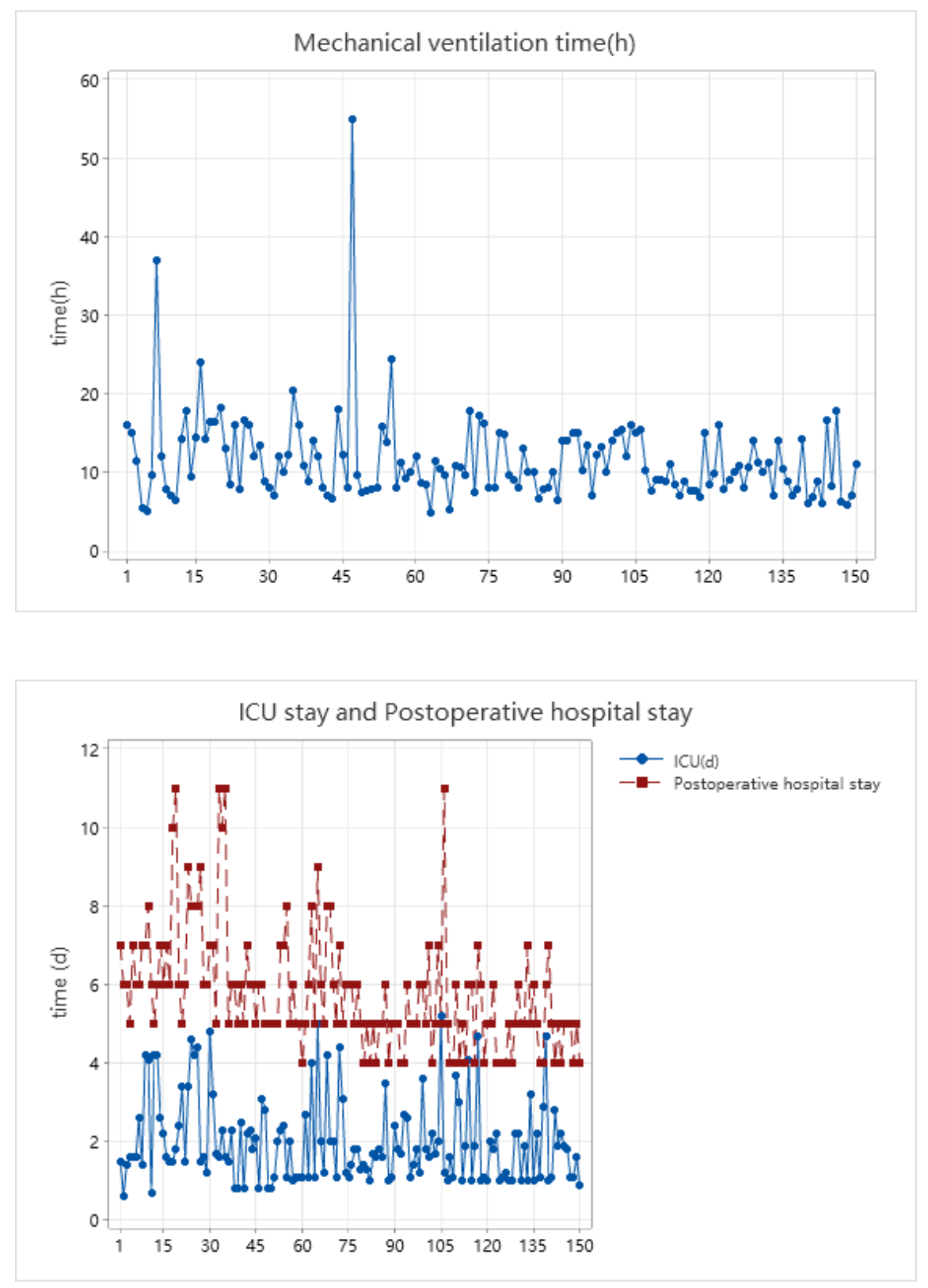

Figure3 Time Series Chart of AC and CPB time(a), postoperative drainage(b), duration of mechanical ventilation(c), ICU stay and postoperative hospital stay(d).

\section{Table}

Table1 Preoperative baseline characteristics

\begin{tabular}{ll}
\hline Variable & Value \\
\hline Male/Female & $93 / 57$ \\
Age & $48.15 \pm 15.875$ \\
Hypertension & 46 \\
Diabetes & 13
\end{tabular}




\begin{tabular}{ll}
\hline Variable & Value \\
\hline Weight & $65.71 \pm 11.94$ \\
Height & $165.61 \pm 8.63$ \\
BMI & $0.24 \pm 0.034$ \\
CAD & 16 \\
AF & 16 \\
EuroScore II & $2.555 \pm 2.44$ \\
Left atrial diameter (LA) & $43.9 \pm 4.84$ \\
Left ventricular end-diastolic diameter (LVDD) & $49.11 \pm 5.241$ \\
Left ventricular ejection fraction (LVEF) & $64.91 \pm 2.938$ \\
NYHA CLASS grade & \\
2 & 37 \\
3 & 86 \\
4 & 27 \\
Cause & \\
Degenerative valve disease & 101 \\
Barlow's disease & 13 \\
Congenital valve disease & 12 \\
Infective endocarditis & 12 \\
Rheumatic valve disease & 10 \\
Others & 2 \\
\hline
\end{tabular}

Table2 Operation and postoperative characteristics

\begin{tabular}{lllll}
\hline Variable & Group1 & Group2 & Group3 & P \\
\hline CPB (min) & $168.82 \pm 23.63$ & $150.94 \pm 18.83$ & $138.86 \pm 17.06$ & ¡0.01* \\
AC (min) & $111.44 \pm 19.943$ & $101.92 \pm 13.124$ & $90.58 \pm 10.433$ & ¡0.01* \\
Postoperative chest drainage in the first 24 h (ML) & $244.4 \pm 114.93$ & $205.4 \pm 95.238$ & $129.8 \pm 41.477$ & ¡ $^{*} .01^{*}$ \\
Mechanical ventilation length (H) & $13.26 \pm 8.179$ & $11.05 \pm 3.709$ & $10.28 \pm 3.297$ & $0.022^{*}$ \\
ICU stay (D) & $2.49 \pm 1.968$ & $2.08 \pm 1.515$ & $1.9 \pm 1.099$ & 0.164 \\
Postoperative hospital stays (D) & $6.64 \pm 1.67527$ & $5.36 \pm 1.00529$ & $5.14 \pm 1.4287$ & $\mathrm{i}^{*} 0.01^{*}$ \\
\hline
\end{tabular}

Table3 Postoperative adverse event

\begin{tabular}{ll}
\hline Variable & value \\
\hline Hospital mortality, n (\%) & 0 \\
Serious complications & 0 \\
Secondary thoracotomy & 0 \\
Respiratory failure, n (\%) & 0 \\
IABP implantation & $2(0.13 \%)$ \\
Delayed extubation & 0 \\
Secondary intubation & 0 \\
Dialysis for Renal Failure & $1(0.6 \%)$ \\
Pleural effusion & 0 \\
Pneumothorax & 0 \\
Cerebral infarction & $1(0.6 \%)$ \\
Cerebral hemorrhage & 0 \\
Pulmonary Infection & 0
\end{tabular}


Variable

Poor healing of incision

Sternal dehiscence value

0 